\title{
Sources et méthodes en histoire des sciences du vivant et de la santé (Moyen Âge, début de l'Époque moderne)
}

Laetitia Loviconi

\section{OpenEdition}

Édition électronique

URL : https://journals.openedition.org/ashp/4694

DOI : 10.4000/ashp.4694

ISSN : 1969-6310

Éditeur

Publications de l'École Pratique des Hautes Études

\section{Édition imprimée}

Date de publication : 1 septembre 2021

Pagination : 363-365

ISSN : 0766-0677

\section{Référence électronique}

Laetitia Loviconi, « Sources et méthodes en histoire des sciences du vivant et de la santé (Moyen Âge, début de l'Époque moderne) ", Annuaire de l'École pratique des hautes études (EPHE), Section des sciences historiques et philologiques [En ligne], 152 | 2021, mis en ligne le 14 juin 2021, consulté le 23 juin 2022. URL : http://journals.openedition.org/ashp/4694 ; DOI : https://doi.org/10.4000/ashp.4694 


\title{
SOURCES ET MÉTHODES EN HISTOIRE DES SCIENCES DU VIVANT ET DE LA SANTÉ (MOYEN ÂGE, DÉBUT DE L'ÉPOQUE MODERNE)
}

\author{
Maître de conférences : $\mathrm{M}^{\mathrm{me}}$ Laetitia LoviconI
}

Programme de l'année 2019-2020 : I. Savoirs physiologiques et res naturales dans les æeuvres de pratique médicale (XIII'-XVI siècle). - II. Les mouvements corporels : théories et controverses (XIII ${ }^{e}-X V I I^{e}$ siècle).

\section{Savoirs physiologiques et res naturales dans les æuvres de pratique médicale (XIII ${ }^{e}-X V I^{e}$ siècle)}

Après avoir analysé le champ disciplinaire de l'histoire des sciences du vivant et de la santé (historiographie et insertion à l'EPHE - séminaires de Mirko Grmek et de Danielle Jacquart notamment), nous avons abordé l'histoire du terme physiologia et celle des savoirs s'y rapportant en la définissant comme étude du fonctionnement corporel considéré dans un état « naturel », à savoir dans un état de santé si l'on s'attache au domaine médical.

Ce n'est qu'au XvI ${ }^{\mathrm{e}}$ siècle que fut rédigé un ouvrage médical intitulé Physiologia. En effet, en 1542, parut le De naturali parte medicinae, une œuvre de Jean Fernel, dans laquelle l'auteur affirme expliquer la constitution du corps humain entièrement sain et dont l'introduction mentionne le terme physiologia pour désigner la branche de la théorie médicale qui étudie « la nature universelle de l'homme », se distinguant de l'étiologie, de la sémiologie, de la diététique et de l'hygiène ainsi que de la thérapeutique. En 1554, c'est justement sous le titre de Physiologia que cet ouvrage fut à nouveau publié, avec une nouvelle introduction dans laquelle Fernel affirme que la physiologie traite « de la nature, des facultés et des fonctions de l'homme quand il est en pleine santé ». Si, au Moyen Âge, le terme physiologia avait été au contraire absent des ouvrages médicaux, il ne faudrait pas pour autant croire qu'aucun savoir ni discours de physiologie médicale n'existait à cette période. Concepts et théories physiologiques étaient en effet exposés et discutés par les médecins médiévaux dans des discussions abordant les composants et les opérations du corps, sous l'appellation de res naturales.

Pour comprendre cette évolution terminologique, nous avons retracé la fortune du mot grec physiologia à travers l'Antiquité, depuis ses utilisations non médicales et médicales dans des ouvrages galéniques et pseudo-galéniques, puis nous avons examiné dans des œuvres médicales ravennates, alexandrines et arabes (dans leurs traductions latines), l'émergence et l'évolution d'une notion renvoyant aux savoirs physiologiques: celle de res naturales. Entre la fin $\mathrm{du} \mathrm{XI}^{\mathrm{e}}$ siècle et le $\mathrm{XIV}^{\mathrm{e}}$ siècle, 
un nombre considérable d'œuvres médicales et à contenu zoo-, bio-physiologiques abordant les res naturales, donc des savoirs physiologiques, a été traduit puis intégré et commenté dans le cadre des enseignements de médecine. On a notamment examiné les modalités d'adaptation, de traduction et d'intégration des œuvres d'Aristote, Galien, Haly Abbas, Johannitius, Avicenne, Averroès. En tenant compte de la période de ces traductions, nous avons ensuite focalisé nos conférences sur les discours physiologiques inclus dans des œuvres écrites entre le $\mathrm{XIII}^{\mathrm{e}}$ siècle et le $\mathrm{XVI}^{\mathrm{e}}$ siècle, en considérant la parution de la Physiologia de Fernel comme terminus a quo. Les œuvres choisies pour examiner ces discours ont été les practicae médicales de cette période, des œuvres constituant un genre développé à partir $\mathrm{du} \mathrm{XI}^{\mathrm{e}}$ siècle et visant à fournir des indications essentielles au praticien en vue de préserver ou restaurer la santé. D'une part, un tel choix permet de déterminer dans quelle mesure le développement d'une médecine savante à cette période s'accompagne d'une attention et d'une exposition croissante de la physiologie. D'autre part, il permet d'explorer quelle pouvait être la place de la physiologie dans l'appréhension et la maîtrise des savoirs relevant de la pathologie et de la thérapeutique, au cœur de la pratique médicale.

Les conférences ont successivement abordé les aspects suivants dans le corpus de practicae:

- Quantification, localisation, utilités, sujets des discours physiologiques;

- Discours sur les éléments, complexions, humeurs;

- Discours sur les membres homéomères;

- Discours sur les membres instrumentaux et les vertus;

- Discours sur la faculté naturelle nutritive, les membres et les opérations associés à cette vertu (digestion, production et devenir des humeurs et des aquosités, assimilation au niveau des membres).

Deux conférences invitées ont eu lieu au cours du premier semestre :

- Charles T. Wolfe : «Le tout et les parties : le cas du vitalisme de Montpellier (XVIII ${ }^{\mathrm{e}}$-XIX ${ }^{\mathrm{e}}$ siècle). Mise en perspective avec la question des parties dans le fonctionnement corporel dans les œuvres de médecine médiévale »;

— Cécilia Bognon-Khuss : «Intussusception, mécanismes vitaux et problème ontologique de la vie au tournant du XVIII ${ }^{\mathrm{e}}$ siècle ».

\section{Les mouvements corporels : théories et controverses (XIII ${ }^{e}-X V I I^{e}$ siècle)}

L'analyse des théories antiques concernant les mouvements respiratoires, cardiaques et pulsatils a été suivie de l'examen des théories médiévales de ces mêmes mouvements, à travers l'étude d'œuvres de Pietro Torrigiano, Pietro d'Abano, Gentile da Foligno, Jacques Despars notamment. L'exploration des théories concernant le mouvement cardiaque a été prolongée sur la période moderne, jusqu'à la fin du $\mathrm{XVII}^{\mathrm{e}}$ siècle, en raison de la parution du De motu cordis de William Harvey, ayant donné lieu à la «première révolution biologique » (expression de Mirko Grmek) et au développement de nouvelles modalités d'étude et d'explication des mouvements cardiaques. Ont ainsi été étudiés les auteurs qui, avant Harvey, ont exposé des données anatomiques ou des théories contraires aux enseignements galéniques, pouvant 
inclure l'idée d'un passage pulmonaire ou d'un mouvement circulaire du sang (Ibn al-Nafis, Michel Servet, Realdo Colombo, Andrea Cesalpino, Giordano Bruno). Le De motu cordis a ensuite fait l'objet d'une lecture analytique abordant les aspects historiques et épistémologiques, avant que soient envisagées les différentes réactions à cette publication (en particulier celles de James Primrose, Jean Riolan fils, Robert Fludd, Pierre Gassendi). Parmi la diversité des théories et approches postérieures au De motu cordis, notre attention s'est également portée sur le mécanisme réductionniste de Descartes, sur les approches iatromécaniques de Borelli et de Baglivi, enfin sur l'animisme de Stahl. 